\title{
Continuously Improving Parametric Modeling with Historical Data on the ICESat-2 Mission
}

\author{
Joseph Krygiel \\ NASA Goddard Space Flight Center \\ 8800 Greenbelt Road \\ Greenbelt, MD 20771 \\ 301-286-5766 \\ Joseph.M.Krygiel@nasa.gov
}

\begin{abstract}
This paper delves into the details of the Joint Confidence Level (JCL) process performed for the Ice, Cloud, and Land Elevation Satellite (ICESat)-2 mission and how past performance was incorporated into subsequent JCL models to enable the project to continuously analyze potential slips to their launch readiness date (LRD). One year prior to the mission Preliminary Review (mPDR), the JCL model development process began. The first model was well received at the mPDR, held on October 10, 2012, and the input received by the Standing Review Board was incorporated into the model for the official data drop for key decision point (KDP)-C.
\end{abstract}

The 70\% JCL results of the October $2012 \mathrm{mPDR}$ model forecast an LRD of February 2017 and associated cost of \$830M. This result in 2012 immediately highlighted potential challenges with the project-planned LRD of July 2016.

The year following the mPDR, the project had sustained a oneyear slip in the LRD due to problematic systems engineering requirement issues which impacted all project subsystems. This slip moved the project planned LRD from July 2016 to July 2017, an additional 5 months beyond the 2012 model's $70 \%$ JCL result for the LRD of February 2017. As the project was quickly approaching the mission Critical Design Review (mCDR), the need for reliable JCL results increased significantly. The project held discussions on the JCL modeling process and focused on the input uncertainty distributions. Specifically, to identify the uncertainty distributions that the 2012 mPDR model would have needed to produce a $70 \%$ LRD result of July 2017. This led the project to compare multiple uncertainty distributions, and ultimately spurred the project to utilize uncertainty distributions that incorporated project past performance and historical data to forecast potential LRD slips.

The revised results, created in 2014 and utilizing the new uncertainty distributions, showed that with $70 \%$ confidence, the ICESat-2 mission would launch in August 2018 with a cost of $\$ 1,044 M$. Today, ICESat-2 is scheduled to launch on September 15,2018 with a project management (PM) agreement value of $\$ 1,056 \mathrm{M}$. This illustrates how a JCL model can be continuously improved to produce valuable results for a project, even in cases of LRD delays.

The primary reason for the ICESat-2 LRD delay is due to a laser failure on the primary instrument. Laser failure was one of the highest risk and uncertainty drivers within the JCL model. The project placed the most risk in this area of the model, and the model further identified the laser as the top risk driver and contributor to the LRD result. This further illustrates how a JCL can be used to predict and quantify possible issues on new technology missions.

\section{TABLE OF CONTENTS}

1. INTRODUCTION ............................................................1

2. BACKGROUND ..........................................................2

3. MODEL OVERVIEW \& ASSUMPTIONS......................2

4. Model Cost \& ANAlysis SChEdule

DEVELOPMENT .2

5. RISK REGISTER \& THREATS.................................4

6. ESTIMATING COST \& SCHEDULE UNCERTAINTY .5

8. MPDR JCL MODEL RESULTS ...............................7

9. SCHEDULE SLIP AND MODEL ADJUSTMENTS .......8

10. MCDR JCL MODEL RESULTS ............................10

11. MCDR RESULTS \& THE ICESAT-2 LAUNCH ....12

12. SUMMARY AND LESSONS LEARNED...................12

APPENDICES ............................................................. 13

A. MPDR RESULTS: RISK ADJUSTED ANNUAL

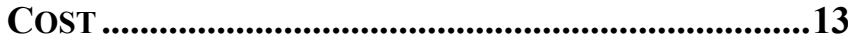

B. MPDR RESULTS: COST S-CURVE.........................13

C. MPDR RESULTS: FINISH DATE S-CURVE..........14

ACKNOWLEDGEMENTS ...........................................14

REFERENCES .........................................................14

BIOGRAPHY ..........................................................

\section{INTRODUCTION}

This paper describes the development of the ICESat-2 JCL between cost and schedule. The first in-house advocate JCL model was developed in October 2012 as was required for $\mathrm{KDP}=\mathrm{C}$.

After sustaining a one-year launch slip in 2013, the project (the ICESat-2 project) had a need for an updated model with reliable results. Finalized in February 2014, the new model incorporated historical data derived from the one-year launch slip. The results of the February 2014 model will then be compared to the current schedule, as of July 2018.

The topics will include an overview of the models, the assumptions made, and the rationale for applying various uncertainties and probabilities. 


\section{BACKGROUND}

Providing a brief overview of the mission, ICESat-2 is a follow-on to the first ICESat mission which launched in 2003 and deorbited in 2010.

ICESat-2 is designed and intended to continue the measurements of sea ice and ice sheets, as well as cloud and land measurements. ICESat-2 is a single instrument mission. That instrument is the Advanced Topographic Laser Altimeter System (ATLAS) and includes a new technology laser. ATLAS was designed, built and tested in-house at the Goddard Space Flight Center (GSFC). ATLAS represents a majority of the total mission life cycle development cost as well as a majority of the project's technical and developmental risk.

\section{Model OVerview \& Assumptions}

The software used to develop the JCL models was Joint Analysis of Cost and Schedule (JACS) which is an add-in to Microsoft (MS) Project. The model includes resources and risks and is identical to the ICESat-2 project Integrated Master Schedule (IMS) in terms of durations and logic.

The project ensured that the cost loading was timely, transparent, traceable, and defendable. The costs were decomposed to time-dependent and time-independent costs. Finally, spending contours were assigned to the costs.

The model contains all risks, threats, and liens. The risks were only placed on the costs to go. The risk probability of occurrence and costs/schedule consequences were identified.
For correlation, we evaluated the nominal values used for space projects, modeled accordingly, and determined a value of 0.5 should be used.

The models included launch vehicle costs and all costs to go, including Phase E, in order to have a full life cycle cost (LCC) comparison between our baseline plan and the model results.

The JCL development process was a very collaborative effort, in which contributions from everyone made for a successful JCL. All team members provided input into the JCL, the project worked extensively with the Standing Review Board, and we received much assistance from the NASA Cost Analysis Division.

\section{Model Cost \& Analysis Schedule DEVELOPMENT}

\subsection{Cost Development}

The model consists of development costs up through and including launch. Costs to date, funded schedule reserve, on orbit checkout, and Phase E costs are included in the results, but are not affected by risks or uncertainty.

For the 2012 mPDR model, actual costs through June 30, 2012 are added to the final JCL results to calculate the total ICESat-2 JCL cost (including Pre-Phase A costs).

The ICESat-2 JCL allocated available budget of $\$ 452 \mathrm{M}$ across schedule elements in phases B, C, and D.

Figure 1 shows the trace from the mission LCC to the JCL value.

\begin{tabular}{|l|r|}
\hline & Value \\
\hline Total LCC (incl. Pre-Phase A) & $\$ 806 \mathrm{M}$ \\
\hline Less Post Launch (JCL finish date) & $\$ 69 \mathrm{M}$ \\
\hline Less Cost-to-Date (6/30/12) & $\$ 195 \mathrm{M}$ \\
\hline Total B/C/D Cost & $\$ 542 \mathrm{M}$ \\
\hline Less Project UFE (Reserve) & $\$ 90 \mathrm{M}$ \\
\hline JCL Input Value & $\$ 452 \mathrm{M}$ \\
\hline
\end{tabular}

Figure 1. Trace from Life-Cycle Cost (LCC) to the JCL value 
Within the analysis model, hammock tasks (schedule summary tasks used to encompass the duration of all work within an area of interest) were created to support cost loading. Then, spending contour profiles were assigned to all costs for how we predicted the cost spending to occur. The subsystem product development leads (PDLs) were interviewed for their assessment of these spending contours, as well as their input on the uncertainty levels to assign (low, medium or high).

Finally, for each hammock task activity, time-independent and time-dependent costs were identified, which translate into approximately 34 schedule activities which were cost loaded.

The time-dependent costs scaled with activity durations, meaning, as activity durations and finish dates push out, due to risk impacts, the time-dependent cost will vary and most likely increase. Conversely, time-independent costs are associated with a specific activity, but do not scale with duration.

Figure 2 provides a high-level, rolled-up view of the entire model, as well as providing insight into the level in which costs were loaded into the analysis schedule.

\begin{tabular}{|c|c|c|c|c|c|c|c|c|c|c|c|c|c|c|c|c|c|c|}
\hline & (i) & Prof-WBS & U1D & - & Start & Finish & Dur & $\%$ & $\begin{array}{l}\text { Total } \\
\text { Slack } \\
\end{array}$ & $\begin{array}{l}J C \\
C_{0}\end{array}$ & $\begin{array}{l}\text { JACS TI } \\
\text { Task Cost } \\
\end{array}$ & $\begin{array}{l}\text { JAC } \\
\text { Thrt } \\
\text { \% } \\
\text { Like }\end{array}$ & $\begin{array}{l}\text { JACS TI Cost } \\
\text { Uncertainty }\end{array}$ & $\begin{array}{l}\text { JACS } \\
\text { TI } \\
\text { pendin } \\
\text { :ontou }\end{array}$ & $\begin{array}{l}\text { JACS TD } \\
\text { Task Cost }\end{array}$ & $\begin{array}{l}\text { JACS TD Cost } \\
\text { Uncertainty }\end{array}$ & $\begin{array}{l}\text { JACS } \\
\text { TD } \\
\text { pendin! } \\
\text { Contour }\end{array}$ & $\begin{array}{l}\text { JACS Duration } \\
\text { Uncertainty }\end{array}$ \\
\hline 0 & & & 0 & - ICESat II Master Schedule & $3 / 30 / 2012$ & $1 / 15 / 2016$ & 889d & $0 \%$ & Od & 0 & $\$ 0.00$ & 0 & & & $\mathbf{5 0 . 0 0}$ & & & \\
\hline 1 & & 883151 & 9201 & - ICESat-2 Total Mission & 6/30/2012 & 1/145/2016 & 889d & $0 \%$ & od & 0 & $\mathbf{\$ 0 . 0 0}$ & 0 & & & $\mathbf{5 0 . 0 0}$ & & & \\
\hline 2 & & 883151.01 & 5496 & - Management & 6/30/2012 & 1/155/2016 & $889 \mathrm{~d}$ & $0 \%$ & Od & 0 & $\mathbf{s 0 . 0 0}$ & 0 & & & $\mathbf{\$ 0 . 0 0}$ & & & \\
\hline 3 & & 883151.01 & 9080 & - Management (Hammock) & 6/30/2012 & 1/15/2016 & $889 \mathrm{~d}$ & $0 \%$ & od & 1 & $\$ 2,234.50$ & 0 & $\operatorname{Tr} \mathbf{r}^{\prime}(95,100,105,10,90)$ & Flat & $\mathbf{s 1 9 , 3 6 3 . 9 0}$ & $\operatorname{Tr}^{\prime}(95,100,105,10,90)$ & Flat & \\
\hline 28 & & 883151.02 & 5510 & - Systems Engineering (GSFC) & 6/30/2012 & 1/156/2016 & 889d & $0 \%$ & od & 0 & $\$ 0.00$ & 0 & & & $\mathbf{\$ 0 . 0 0}$ & & & \\
\hline 29 & & 883151.02 & 9117 & - Systems Engineering (Hammock) & 6/30/2012 & 1/144/2016 & 888d & $0 \%$ & od & 1 & $\$ 2,100.00$ & 0 & $\operatorname{Tr}(95,100,105,10,90)$ & Flat & $\$ 7,937.20$ & Tri'(95,100,105,10,90) & Flat & \\
\hline 40 & & 883151.03 & 5526 & - Safety and Mission Assurance & 6/30/2012 & 1/15/2016 & $889 \mathrm{~d}$ & $0 \%$ & od & 0 & $\$ 0.00$ & 0 & & & $\$ 0.00$ & & & \\
\hline 41 & & 883151.03 & 9120 & - Safety and Mission Assurance (Hammock) & 6/30/2012 & 1/15:/2016 & 889d & $0 \%$ & od & 1 & $\$ 0.00$ & & $\operatorname{Tr}(95,100,105,10,90)$ & Flat & $\$ 7,122.50$ & Tri' $(95,100,105,10,90)$ & Flat & \\
\hline 57 & & 883151.05 & 5524 & $\begin{array}{l}\text { - Science Payload (GSFC ATLAS Instrument } \\
\text { Development) }\end{array}$ & 6/30/2012 & 2/1312015 & 658d & $0 \%$ & od & 0 & $\$ 0.00$ & 0 & & & $\mathbf{s 0 . 0 0}$ & & & \\
\hline 58 & & 883151.05 .01 & 8794 & - ATLAS GSFC Instrument Management (Hammock) & 6/30/2012 & 2/13/2015 & 658d & $0 \%$ & 231d & 1 & $\mathbf{s 2 8 . 0 0}$ & 0 & $\operatorname{Trr}^{\prime}(95,100,105,10,90)$ & Flat & $\$ 4,353.80$ & Tri' $(95,100,105,10,90)$ & Flat & \\
\hline 61 & & 883151.05 .02 & 8795 & - Instrument Systems Engineering & 6/30/2012 & 2/13/2015 & $658 \mathrm{~d}$ & $0 \%$ & 231d & 1 & \$o.00 & 0 & Tri'(95,100,105,10,90) & Flat & $\$ 7,023.90$ & Tri'(95, 100,105,10,90) & Flat & \\
\hline 64 & & 883151.05 .05 & 8959 & - Instrument Development & 6/30/2012 & 2/13/2015 & $658 \mathrm{~d}$ & $0 \%$ & od & 0 & $\$ 0.00$ & 0 & & & $\mathbf{s} .00$ & & & \\
\hline 65 & & 883151.05 .05 .01 & 8797 & - Optics (Hammock) & 6/30/2012 & 11/13/2013 & $345 \mathrm{~d}$ & $0 \%$ & 544d & 1 & $\$ 3,919.00$ & 0 & $\operatorname{Tr}(95,105,110,10,90)$ & D) Turtle & $\$ 10,098.70$ & $\operatorname{Trr}(95,100,110,10,90)$ & ) it Loaded & \\
\hline 69 & & 883151.05 .05 .02 & 8798 & - Mechanisms & 6/30/2012 & 6/26/2013 & $249 d$ & $0 \%$ & $640 \mathrm{~d}$ & 1 & $\$ 1,984,00$ & 0 & $\operatorname{Tr}(95,105,110,10,90)$ & D) ILoaded & $\$ 1,762.00$ & Tri'(95, 100,105,10,90) & i) it Loaded & \\
\hline 73 & & & 9330 & - Primary Structure & 6/30/2012 & 6/13/2013 & $240 \mathrm{~d}$ & $0 \%$ & 649d & 1 & $\$ 3,058.00$ & 0 & $\operatorname{Tr}(95,100,105,10,90)$ & II Turtle & $\$ 1,689.00$ & $\operatorname{Tr}(95,100,105,10,90)$ & i) it Loaded & \\
\hline 77 & & 883151.05 .05 .03 & 8799 & $\begin{array}{l}\text { - Laser Transmitter Integrated Product (through } \\
\text { SM2 \& SN3) (Hammock) }\end{array}$ & 6/30/2012 & 12/18/2013 & $369 \mathrm{~d}$ & $0 \%$ & $520 \mathrm{~d}$ & 1 & $\$ 1,176.00$ & 0 & $\operatorname{Trr}^{\prime}(95,105,110,10,90)$ & $\begin{aligned} \text { Front } \\
\text { Loaded }\end{aligned}$ & $\$ 20,047.00$ & $\operatorname{Tr}(95,100,105,10,90)$ & $\begin{array}{r}\text { Front } \\
\text { Loaded }\end{array}$ & \\
\hline 81 & & 883151.05 .05 .04 & 8800 & - Laser Reference System (LRS) (Hammock) & 6/30/2012 & 11/14/2013 & $346 \mathrm{~d}$ & $0 \%$ & 543d & 1 & $\$ 1,700.00$ & 0 & $\operatorname{Tr}(95,100,105,10,90)$ & I)! Loaded & $\$ 2,400.00$ & Tri' $(95,100,105,10,90)$ & i) it Loaded & \\
\hline 85 & & 883151.05 .05 .05 & 8801 & - Thermal (Hammock) & 6/30/2012 & 8/30/2013 & 295d & $0 \%$ & $594 d$ & 1 & $\$ 2,415.00$ & 0 & $\operatorname{Tr}(95,100,105,10,90)$ & n! Loaded & $\$ 1,363.00$ & $\operatorname{Tr}^{\prime}(95,100,105,10,90)$ & 1) Flat & \\
\hline 89 & & 883151.05 .05 .06 & 8802 & - MEB (Hammock) & 6/30/2012 & 10/1/2013 & $316 \mathrm{~d}$ & $0 \%$ & $573 \mathrm{~d}$ & 1 & $\$ 5,267.00$ & 0 & $\operatorname{Tr}(95,105,110,10,90)$ & D) Loaded & $\$ 11,952.90$ & Trir $(95,100,105,10,90)$ & 1) it Loaded & \\
\hline 95 & & 883151.05 .05 .07 & 8803 & - Flight Software (Hammock) & 6/30/2012 & 7/145/2013 & 261d & $0 \%$ & $628 \mathrm{~d}$ & 1 & $\$ 245.00$ & 0 & $\operatorname{Tr}(95,100,105,10,90)$ & i) Flat & $\$ 2,128.00$ & $\operatorname{Tr}(95,100,105,10,90)$ & 1) Flat & \\
\hline 99 & & 883151.05 .05 .08 & 8804 & - Detectors (Hammock) & 6/30/2012 & 9/10/2013 & 301d & $0 \%$ & 588d & 1 & \$4,491.00 & 0 & $\operatorname{Tr}(95,105,110,10.90)$ & 3) ! Loaded & $\$ 4,359.00$ & $\operatorname{Tr}(95,100,105,10,90)$ & 1) it Loaded & \\
\hline 103 & & 883151.05 .05 .10 & 8806 & - BCE (Hammock) & 6/30/2012 & 11/20/2013 & $350 \mathrm{~d}$ & $0 \%$ & 539d & 1 & $\$ 1,240.00$ & 0 & $\operatorname{Tr}(95,100,105,10,90)$ & II Flat & $\$ 1,523.00$ & Trir $95,100,105,10,90)$ & 11 Flat & \\
\hline 107 & & 883151.05 .05 .11 & 8807 & - Flight Science Algorithms (Hammock) & 6/30/2012 & 6/28/2013 & 251d & $0 \%$ & $638 \mathrm{~d}$ & 1 & $\$ 0.00$ & 0 & & & $\$ 1,057.00$ & $\operatorname{Tr}(95,100,105,10,90)$ & Flat & \\
\hline 111 & & 883151.05 .05 .12 & 8808 & - Power Systems (Hammock) & 6/30/2012 & 9/18/2013 & 307d & $0 \%$ & $582 \mathrm{~d}$ & 1 & $\$ 2,919.00$ & 0 & $\operatorname{Tr}(95,105,110,10,90)$ & D) ! Loaded & $\$ 2,194.00$ & $\operatorname{Tr}(95,100,105,10,90)$ & i) it Loaded & \\
\hline 115 & & 883151.05 .05 .16 & 8809 & - Detector Array Assembly (Hammock) & 6/30/2012 & $1 / 2 / 2014$ & $378 \mathrm{~d}$ & $0 \%$ & 511d & 1 & $\$ 643.00$ & 0 & $\operatorname{Tr}(95,100,105,10,90)$ & 1)! Loaded & $\$ 1,019.00$ & $\operatorname{Tr}(95,100,105,10,90)$ & i) it Loaded & \\
\hline 121 & & 883151.05 .05 .17 & 8810 & - Optical Fitter Assembly (Hammock) & 6/30/2012 & $12 / 13 / 2013$ & $366 \mathrm{~d}$ & $0 \%$ & $523 \mathrm{~d}$ & 1 & $\$ 1,826.00$ & 0 & $\operatorname{Tr}(95,105,110,10,90)$ & ) ILoaded & $\$ 1,780.00$ & $\operatorname{Tri}(95,100,105,10,90)$ & 1) :arly Peak & \\
\hline 125 & & 883151.05 .05 .18 & 9333 & - Materials Eng (Hammock) & 6/30/2012 & 2/24/2014 & 413d & $0 \%$ & $476 \mathrm{~d}$ & 1 & $\$ 0.00$ & 0 & & & $\mathbf{\$ 5 0 9 . 0 0}$ & $\operatorname{Tr}(95,100,105,10,90)$ & 1) Flat & \\
\hline 129 & & 883151.05 .05 .19 & 9204 & - OOS (Hammock) & 6/30/2012 & $12 \pi / 2012$ & 111d & $0 \%$ & $778 \mathrm{~d}$ & 1 & $\$ 52.00$ & 0 & $\operatorname{Tr} \operatorname{ri}^{\prime}(95,400,105,10,90)$ & Flat & $\mathbf{s 3 7 3 . 0 0}$ & $\operatorname{Tri}(95,100,105,10,90)$ & Flat & \\
\hline 133 & & 883151.05 .05 .21 & 9205 & - FLATLAS (Hammock) & 6/30/2012 & $5 / 31 / 2013$ & $231 d$ & $0 \%$ & 658d & 1 & $\$ 31.00$ & 0 & $\operatorname{Tr}(95,100,105,10,90)$ & II Flat & $\$ 146.00$ & Tri'(95, 100,105,10,90) & & \\
\hline 137 & & 883151.05 .05 .22 & 9208 & - Harness (Hammock) & 6/30/2012 & 4/166/2013 & 199d & $0 \%$ & 690d & 1 & $\$ 232.00$ & 0 & $\operatorname{Tr}(95,105,110,10,90)$ & D) irly Peak & $\$ 883.00$ & $\operatorname{Irr}(95,100,105,10,90)$ & II) Late Peak & \\
\hline 141 & & 883151.05 .05 .23 & 9220 & - Parts Mgmt & 6/30/2012 & $2 / 25 / 2013$ & 163d & $0 \%$ & $726 \mathrm{~d}$ & 1 & $\$ 1,080.00$ & 0 & $\operatorname{Tr}(95,100,105,10,90)$ & in Flat & $\$ 0.00$ & & & \\
\hline 145 & & 883151.05 .05 .24 & 9211 & - TAMS (Hammock) & 6/30/2012 & 10/1/2013 & $316 \mathrm{~d}$ & $0 \%$ & $573 \mathrm{~d}$ & 1 & $\$ 242.00$ & 0 & $\operatorname{Tr}(95,100,105,10,90)$ & 1) :Loaded & $\$ 645.00$ & $\operatorname{Tr}^{\prime}(95,100,105,10,90)$ & I) arly Peak & \\
\hline 149 & & 883151.05 .05 .25 & 9214 & - Optical Fibers (Hammock) & 6/30/2012 & $5 / 142013$ & $219 d$ & $0 \%$ & $670 \mathrm{~d}$ & 1 & $\$ 27.00$ & 0 & $\operatorname{Tr}(95,105,110,10,90)$ & D) Flat & $\$ 700.00$ & Tri'(95, 100,105,10,90) & 1) Flat & \\
\hline 153 & & 883151.05 .05 .26 & 9217 & - AMCS (Hammock) & 6/30/2012 & 10/4/2013 & $316 \mathrm{~d}$ & $0 \%$ & $573 \mathrm{~d}$ & 1 & $\$ 50.00$ & 0 & $\operatorname{Tr}(95,100,105,10,90)$ & Flat & $\$ 1,376.00$ & $\operatorname{Tr}(95,100,105,10,90)$ & Flat & \\
\hline 157 & & 883151.05 .05 .09 & 8811 & - ATLASI\&T & 6/3/2013 & $2 / 13 / 2015$ & $427 \mathrm{~d}$ & $0 \%$ & $64 d$ & 0 & $\mathbf{s 0 . 0 0}$ & 0 & & & $\$ 0.00$ & & & \\
\hline 158 & & 883151.05 .05 .09 & 9123 & - ATLAS I8T (Hammock) & 6/14/2013 & 2/133/2015 & $418 \mathrm{~d}$ & $0 \%$ & 231d & 1 & $\$ 1,933.00$ & 0 & $\operatorname{Tr}(95,105,110,10,90)$ & D) ate Peak & $\$ 23,616.00$ & $\operatorname{Tr}(95,100,110,10,90)$ & D) Late Peak & \\
\hline 189 & & 883151.06 & 755 & - Spacecraft Development & 6/30/2012 & 10/28/2014 & $585 \mathrm{~d}$ & $0 \%$ & od & 0 & $\$ 0.00$ & 0 & & & $\$ 0.00$ & & & \\
\hline 190 & & 883151.06 & 8793 & - Spacecratt Management (OSC TD Work) & 6/30/2012 & 10/28:2014 & $585 \mathrm{~d}$ & $0 \%$ & od & 0 & $\$ 0.00$ & 0 & & & 50.00 & & & \\
\hline 191 & 0 & 883151.06 & 9132 & - Spacecratt Management (OSC TD Work) (Hamme & 6/30/2012 & 10/23/2014 & 585d & $0 \%$ & od & 1 & $\$ 95,468.90$ & 0 & $\operatorname{Tr}(95,105,110,10,90)$ & Turtle & 50.00 & & & \\
\hline 202 & & 883151.06 & 8836 & $\begin{array}{l}\text { - Spacecraft Systems Engineering (LOE) (In House } \\
\text { TO Work\} }\end{array}$ & 6/30/2012 & 10/28/2014 & $585 \mathrm{~d}$ & $0 \%$ & od & 0 & $\$ 0.00$ & 0 & & & $\mathbf{s 0 . 0 0}$ & & & \\
\hline 203 & $\theta$ & 883151.06 & 9129 & $\begin{array}{l}\text { - Spacecraft Systems Engineering (LOE) (ln } \\
\text { House TD Work) (Hammock) }\end{array}$ & 6/30/2012 & 10/28:2014 & 585d & $0 \%$ & od & 1 & $\$ 0.00$ & 0 & & & $\$ 2,057.00$ & $\operatorname{Tr}(95,100,105,10,90)$ & Turtle & \\
\hline 215 & & 883151.08 & 5678 & - Launch Vehicle (Procurement) & 6/30/2012 & 10/9/2015 & $824 d$ & $0 \%$ & od & 0 & $\$ 0.00$ & 0 & & & 50.00 & & & \\
\hline
\end{tabular}

Figure 2. Mapping of Cost Elements to Schedule Activity

\subsection{Analysis Schedule Development}

The following section will capture the pertinent steps involved with the development of the JCL Analysis Schedule.

The ICESat-2 IMS is a fully detailed schedule, from start to finish, for each subsystem. The Analysis Schedule was built directly from the IMS detail. The Analysis Schedule was constructed at a level of detail that effectively supports cost loading and risk integration. Moreover, the project allowed for the risks to dictate the level of detail within the Analysis Schedule. The durations used in the Analysis Schedule encompass the duration of the entire development of their respective subsystems. Approximately 300 activities are included in the model.

All pertinent IMS hand-offs between subsystems are reflected within the JCL model. Use of the unique identifier (UID) within the ICESat-2 IMS ensures the model accurately reflects the finish dates and slack within the IMS, as can be seen in Figure 3. Great attention to detail ensured correct slack for each of the subsystem deliveries was correctly captured within the model. The Analysis Schedule was set up with "as-soon-as-possible" tasks with no constraints.

The activities in the mPDR model began on June 30, 2012 and continued through the LRD at the time, July 2016. 

zero days of duration.

\begin{tabular}{|c|c|c|c|c|c|c|c|c|c|c|c|c|c|c|c|c|c|}
\hline Proj-WBS & Notes & UD & JCL Schedule Mod & lel. & Start & Finish & Dur & . & $\begin{array}{l}\text { Total } \\
\text { Slack }\end{array}$ & $\begin{array}{l}\text { JACS TD } \\
\text { Task } \\
\text { Cost } \\
\end{array}$ & $\begin{array}{l}\text { AC: } \\
\text { TD } \\
\text { end } \\
\text { into }\end{array}$ & $\begin{aligned} \text { JACS } \\
\text { Uno }\end{aligned}$ & $\begin{array}{l}\text { To Cost } \\
\text { ittainty }\end{array}$ & $\begin{array}{r}\text { JAA } \\
\text { TIT } \\
\text { To }\end{array}$ & $\begin{array}{l}\text { cs } \\
\text { ask } \\
\text { sst }\end{array}$ & & \\
\hline 883151.05 .05 .02 & & 8798 & + Instrument Mechanical Systen [ & Unique & Task Name & & & & & & & & $\%$ & Dur. & Start & Finish & Slack \\
\hline 883151.05 .05 .02 .01 & UID 130028114 & 9016 & Mechanisms & 10. & & & ATL & $\mathbf{A S}$ & IMS & & & $\checkmark$ & r & 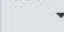 & & $r$ & \\
\hline 883151.05 .05 .02 .02 & UID 130028123 & 9017 & Primary Structure & & & & & & & & & & & & & & \\
\hline 883151.05 .05 .03 & & 8799 & - Laser Transmitter Integrated & 12586040 & & - FM MEB & & & & & & & $0 \%$ & $201 \mathrm{~d}$ & 12/13/20 & $1210 / 1 / 2013$ & $129 \mathrm{~d}$ \\
\hline & & & (through SN2 \& SN3) (Hammoch & 12586041 & & - MEB FM & 11 Comp & onent: & its Integration & $\mathrm{n}$ and Fit Chec & & & $0 \%$ & $164 \mathrm{~d}$ & $12 / 13 / 20$ & $128 / 8 / 2013$ & od \\
\hline 883151.05 .05 .03 & & 9168 & Laser - Start for Hammock & 12586051 & & (Rec) & MEB FM & $11 \mathrm{SBC}$ & CFM1 & & & & $0 \%$ & od & $12 / 13 / 20$ & $12 \quad 12 / 13 / 2012$ & $129 \mathrm{~d}$ \\
\hline 883151.05 .05 .03 & & 9169 & Laser - Finish for Hammock & 12586043 & & $(\operatorname{Rec})$ & MEB FM & 11 Mech & chanical Enclosu & & & & $0 \%$ & od & 3/6/201 & $13 \quad 3 / 6 / 2013$ & $74 \mathrm{~d}$ \\
\hline 883151.05 .05 .03 & UID 130028117 & 9190 & Laser Development & 12586044 & & $(\operatorname{Rec})$ & MEB FM & $11 \mathrm{BP} / \mathrm{H}$ & HWFM1 & & & & $0 \%$ & $0 \mathrm{~d}$ & $5 / 3 / 201$ & $5 / 3 / 2013$ & $32 \mathrm{~d}$ \\
\hline 883151.05 .05 .04 & & 8800 & - Laser Reference System (LRS) & 12586046 & & (Rec) & MEB FM & 11 MCE & EFM1 & & & & $0 \%$ & od & 3/20/20 & 3/20/2013 & $96 \mathrm{~d}$ \\
\hline & & & (Hammock) & 12586047 & & $(\operatorname{Rec})$ & MEB FM & 11 нКт & TFM1 & & & & $0 \%$ & od & $7 / 12 / 20$ & $137 / 12 / 2013$ & $16 d$ \\
\hline 883151.05 .05 .04 & & 9170 & LRS - Start for Hammock & 12586053 & & (Rec) & MEB FM & 11 usoc & OC FM1 & & & & $0 \%$ & od & $6 / 28 / 20$ & $136 / 28 / 2013$ & $25 d$ \\
\hline 883151.05 .05 .04 & & 9171 & LRS - Finish for Hammock & 12586049 & & $(\operatorname{Rec})$ & MEB FM & 11 ASC & CFM1 & & & & $0 \%$ & od & 7/5/201 & $7 / 5 / 2013$ & $21 \mathrm{~d}$ \\
\hline 883151.05 .05 .04 & UID 130028130 & 9191 & LRS Development & 12586045 & & $(\operatorname{Rec})$ & MEB FM & 11 PCE 1 & $1 \mathrm{FM}$ & & & & $0 \%$ & od & 7/5/201 & $7 / 5 / 2013$ & $21 \mathrm{~d}$ \\
\hline 883151.05 .05 .05 & & 8801 & - Thermal (Hammock) & 12590683 & & (Rec) & MEB FM & 11 PCE2 & $2 \mathrm{FM}$ & & & & $0 \%$ & od & 8/5/201 & $8 / 5 / 2013$ & od \\
\hline 883151.05 .05 .05 & & 9172 & Thermal - Start for Hammock & 12500684 & & $(\operatorname{Rec})$ & MEB FM & 11 PCE3 & $3 \mathrm{FM}$ & & & & $0 \%$ & od & 8/5/201 & $8 / 52013$ & \\
\hline 883151.05 .05 .05 & & 9173 & Thermal - Finish for Hampeck & 12585496 & $\underline{D}$ & (Rec) & FM1 MEE & BLLPC & PC from PDU & & & & $0 \%$ & od & 4/10/20 & 4/10/2013 & $81 \mathrm{~d}$ \\
\hline 883151.05 .05 .05 & UID 130037475 & 9192 & Thermal Development & & 9/30/2011 & $8 / 30 / 2013$ & $482 d$ & $0 \%$ & $172 d$ & $\$ 0.00$ & & & & & 50.00 & & \\
\hline 883151.05 .05 .06 & & 8802 & - MEB (Hammóck) & & 9/30/2011 & 10/1/2013 & $503 d$ & $0 \%$ & $598 \mathrm{~d}$ & $\$ 8,538.50$ & & & & & $681.4 n$ & & \\
\hline 883151.05 .05 .06 & & 9174 & _. - TRÉB - Start for Hammock & & 9/30/2011 & 9/30/2011 & od & $0 \%$ & 1101d & .50 .00 & & & & & $\$ 0.00$ & & \\
\hline 883151.05 .05 .06 & & 9175 & MEB - Finish for Hammock & & $10 / 1 / 2013$ & 10/1/2013 & od & $0 \%$ & $\ldots \ldots-598 d$ & so.00 & & & & & $\$ 0.00$ & & \\
\hline 883151.05 .05 .06 & UID 130028127 & $9193,-$ & MEB Development & & 9/30/2011 & 10/1/2013 & $503 \mathrm{~d}^{-}$ & $0 \%$ & $38 \mathrm{~d}$ & 50.00 & & & & & s0.00 & & \\
\hline 883151.05 .05 .06 & UID 12585502 & -9227 & MCE to MEB I\&T & & $3 / 2012013$ & $47 / 10 / 2013$ & $79 \mathrm{~d}$ & $0 \%$ & and & $4--30.00$ & & & & & so.00 & & \\
\hline 883151.05 .05 .06 & UID 12585496 & 9229 & LVPC to MEB I\&T & & 4/10/2013 & $7 / 31 / 2013$ & $79 \mathrm{~d}$ & $0 \%$ & 81d) & ) 50.00 & & & & & 50.00 & & \\
\hline 883151.05 .05 .07 & & 8803 & - Flight Software (Hammock) & & 9/30/2011 & 7/15/2013 & 448d & $0 \%$ & $653 d$ & $\$ 3,637.10$ & Flat & & & & $\$ 98.80$ & & \\
\hline 883151.05 .05 .07 & & 9176 & Flight Software - Start for Hamm & & 9/30/2011 & 9/30/2011 & od & $0 \%$ & 1101d & $\$ 0.00$ & & & & & $\$ 0.00$ & & \\
\hline 883151.05 .05 .07 & & 9177 & Flight Software - Finish for Hamn & nock & 7/15/2013 & $7 / 15 / 2013$ & od & $0 \%$ & $653 d$ & $\$ 0.00$ & & & & & $\$ 0.00$ & & \\
\hline 883151.05 .05 .07 & UID 130028099 & 9194 & Flight Software Development & & 9/30/2011 & $7 / 15 / 2013$ & $448 d$ & $0 \%$ & $89 \mathrm{~d}$ & $\$ 0.00$ & & & & & $\$ 0.00$ & & \\
\hline
\end{tabular}

Figure 3. Analysis Schedule Development from IMS

\section{RISK REgISTER \& THREATS}

The ICESat-2 Risk Register is included within the analysis schedule, in a dedicated section beneath the model. Comprised of approximately 70 risks, this list was taken straight from the ICESat-2 risk database. These risks were linked into the analysis schedule as activities using predecessor and successor relationships, but with zero days' duration.

The subsystem PDLs were interviewed for their assessment of the risks, which were quantified in terms of likelihood of occurrence, as well as cost and schedule consequences. Schedule and cost impacts were provided as three-point estimates: low, medium, or high.

The PDLs were asked to characterize the total risk impact beyond the current time-dependent costs (overtime, double shifts, additional parts and materials, additional tests, etc.).

Inputs and assumptions were reviewed by management and the systems engineering team for consistency and accuracy.

Risks were quantified in terms of likelihood of occurrence (\%), schedule consequence (work days), and cost consequence (\$). Project issues and liens, not associated with a discrete risk, were included in the model as additional entries in the risk register or accounted for by applying greater uncertainty to those subsystems which would be impacted, if the risk were realized.

Post-mitigation "Performance risks" were included in the model, but had no impact to cost or schedule.
Figure 4 provides a visual of a small subset of the Risk Register, as it was in 2012 for the mPDR. These top risks focused on the ATLAS hardware and development schedules:

\section{Top Risks}

\begin{tabular}{|c|c|c|c|}
\hline $\begin{array}{l}\text { Rank/ } \\
\text { Trend }\end{array}$ & ID & Title & Approach \\
\hline 1 & $\begin{array}{l}\text { ICESat-2 -Risk- } \\
\text { INSTR-0080 }\end{array}$ & Laser Diode Reliability & Mitigate \\
\hline 2 & $\begin{array}{l}\text { ICESat-2 -Risk- } \\
\text { MISSION-0042 }\end{array}$ & $\begin{array}{l}\text { Daily Data Volume Modeling } \\
\text { Uncertainty }\end{array}$ & Mitigate \\
\hline 3 & $\begin{array}{l}\text { ICESat-2-Risk- } \\
\text { SPACE-0107 }\end{array}$ & $\begin{array}{l}\text { Fault Management Process } \\
\text { on } \\
\text { Spacecraft }\end{array}$ & Mitigate \\
\hline 4 & $\begin{array}{l}\text { ICESat-2-Risk- } \\
\text { SPACE-0108 }\end{array}$ & $\begin{array}{l}\text { Spacecraft Component } \\
\text { Supplier Performance }\end{array}$ & Mitigate \\
\hline 5 & $\begin{array}{l}\text { ICESat-2-Risk- } \\
\text { INSTR-0043 }\end{array}$ & $\begin{array}{l}\text { Uncorrectable On-Orbit } \\
\text { Alignment Elements }\end{array}$ & Watch \\
\hline 6 & $\begin{array}{l}\text { ICESat-2 -Risk- } \\
\text { INSTR-0079 }\end{array}$ & $\begin{array}{l}\text { Optical Damage in Frequency } \\
\text { Doubling Crystal }\end{array}$ & Mitigate \\
\hline 7 & $\begin{array}{l}\text { ICESat-2-Risk- } \\
\text { INSTR-0100 }\end{array}$ & $\begin{array}{l}\text { Flight Laser Build in Parallel } \\
\text { with qualification laser Life } \\
\text { Testing }\end{array}$ & Mitigate \\
\hline 8 & $\begin{array}{l}\text { ICESat-2-Risk- } \\
\text { MISSION-0002 }\end{array}$ & $\begin{array}{l}\text { Undefined SC to Launch } \\
\text { Vehicle; Technical Interface } \\
\text { Uncertainties }\end{array}$ & Mitigate \\
\hline
\end{tabular}

Figure 4. 2012 mPDR Top Risks from the Risk Register 


\section{Estimating Cost \& Schedule UNCERTAINTY}

\subsection{Cost Uncertainty}

All estimated costs have some level of uncertainty. The cost uncertainties are further broken down based on the timedependent and time-independent nature of the costs. Time dependent costs scale with activity durations, whereas time independent costs are associated with a specific activity and do not scale with duration.
The project broke down uncertainty into three levels:

- Low (green) $10 \%$

- Medium (yellow) $20 \%$

- High (red) $30 \%$

The table in Figure 5 shows how we quantified uncertainty ranges: Low, Medium, and High.

\begin{tabular}{|c|c|c|c|}
\hline \multicolumn{4}{|c|}{ Cost Uncertainty (Time Dependent Costs) } \\
\hline & $\begin{array}{c}\text { Green }(95 \%, 100 \% 105 \% \\
\text { variance) }\end{array}$ & $\begin{array}{c}\text { Yellow (98\%, 110\%, 120\% } \\
\text { variance) }\end{array}$ & $\begin{array}{c}\text { Red }(100 \%, 120 \%, 135 \% \\
\text { variance) }\end{array}$ \\
\hline Cost Confidence & $\begin{array}{l}\text { Confident in meeting the } \\
\text { required cost cap. Have } \\
\text { ample margin and overall a } \\
\text { low cost risk. }\end{array}$ & $\begin{array}{l}\text { Little confidence in meeting } \\
\text { the required cost cap. Have } \\
\text { little margin and overall a } \\
\text { risky cost estimate. }\end{array}$ & $\begin{array}{l}\text { Not very confident in } \\
\text { meeting the required cost } \\
\text { cap. No margin and overall } \\
\text { a high risk cost estimate. }\end{array}$ \\
\hline \multicolumn{4}{|c|}{ Cost Uncertainty (Time Independent Costs) } \\
\hline & $\begin{array}{c}\text { Green }(90 \%, 100 \% 110 \% \\
\text { variance) }\end{array}$ & $\begin{array}{c}\text { Yellow (95\%, } 100 \%, 135 \% \\
\text { variance) }\end{array}$ & $\begin{array}{c}\text { Red (99 \%, 100\%, } 155 \% \\
\text { variance) }\end{array}$ \\
\hline Cost Confidence & $\begin{array}{l}\text { Confident in meeting the } \\
\text { required cost cap. Have } \\
\text { ample margin and overall a } \\
\text { low cost risk. }\end{array}$ & $\begin{array}{l}\text { Little confidence in meeting } \\
\text { the required cost cap. Have } \\
\text { little margin and overall a } \\
\text { risky cost estimate. }\end{array}$ & $\begin{array}{l}\text { Not very confident in } \\
\text { meeting the required cost } \\
\text { cap. No margin and overall } \\
\text { a high risk cost estimate. }\end{array}$ \\
\hline
\end{tabular}

Figure 5. 2012 mPDR Cost Uncertainties

These cost uncertainty ratings were based on the opinion of the PDLs and task complexity. These uncertainties were reviewed by the management team to ensure uncertainty ratings were applied uniformly and consistently across the mission elements. Finally, these cost uncertainty distributions are in-line with historical JCL models.

\subsection{Duration Uncertainty}

All planned durations have some level of uncertainty.
Similar to cost uncertainty, we developed these three levels of duration uncertainty and applied them to the subsystems according to discussions with the PDLs. We did not load duration uncertainty to level-of-effort (LOE) tasks.

Similarly to the cost uncertainty, we developed these three levels of duration uncertainty, which can be seen in Figure 6, and applied them to the subsystems according to discussions with the PDLs.

\begin{tabular}{|l|l|l|l|}
\hline \multicolumn{4}{|c|}{ Duration Uncertainty } \\
\hline & \multicolumn{1}{|c|}{ Green (90-100-110) } & \multicolumn{1}{|c|}{ Yellow (95-100-135) } & \multicolumn{1}{c|}{ Red (99-100-155) } \\
\hline Schedule Confidence & $\begin{array}{l}\text { Confident in meeting the } \\
\text { required delivery date. } \\
\text { Have ample margin and } \\
\text { overall a low risk schedule. }\end{array}$ & $\begin{array}{l}\text { Little confidence in meeting the } \\
\text { required delivery date. Have little } \\
\text { margin and overall a risky } \\
\text { schedule. }\end{array}$ & $\begin{array}{l}\text { Not very confident in meeting the } \\
\text { required delivery date. No margin } \\
\text { and overall a high risk schedule. }\end{array}$ \\
\hline
\end{tabular}

\section{Figure 6. 2012 mPDR Duration Uncertainties}

In developing the uncertainties, we reviewed data from the NASA Cost Analysis Data Requirement (CADRe), referenced other Goddard projects, reviewed literature on developing uncertainty ranges, and utilized input received from members of the NASA Cost Analysis Division.
The project made sure not to apply excessive duration uncertainty to tasks that already had an associated risk that impacted duration. The uncertainty applied to tasks were broadly based on the complexity of the work, slack assumptions, and confidence in meeting the plan. 


\subsection{Uncertainty Definition Development}

In an attempt to allow the 2012 mPDR model to be as conservative as possible, with respect to high uncertainty ratings, the project held multiple discussions regarding uncertainty distributions.

Figure 7 is an example of standard triangular distribution for a high uncertainty, showing the $30 \%$ chance of either falling above or below the nominal value.

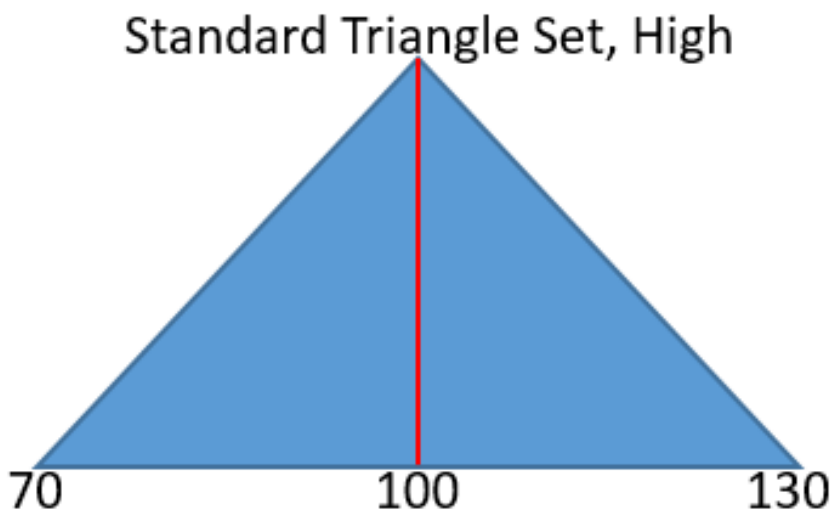

Figure 7. Standard Triangular Distribution for High Uncertainty

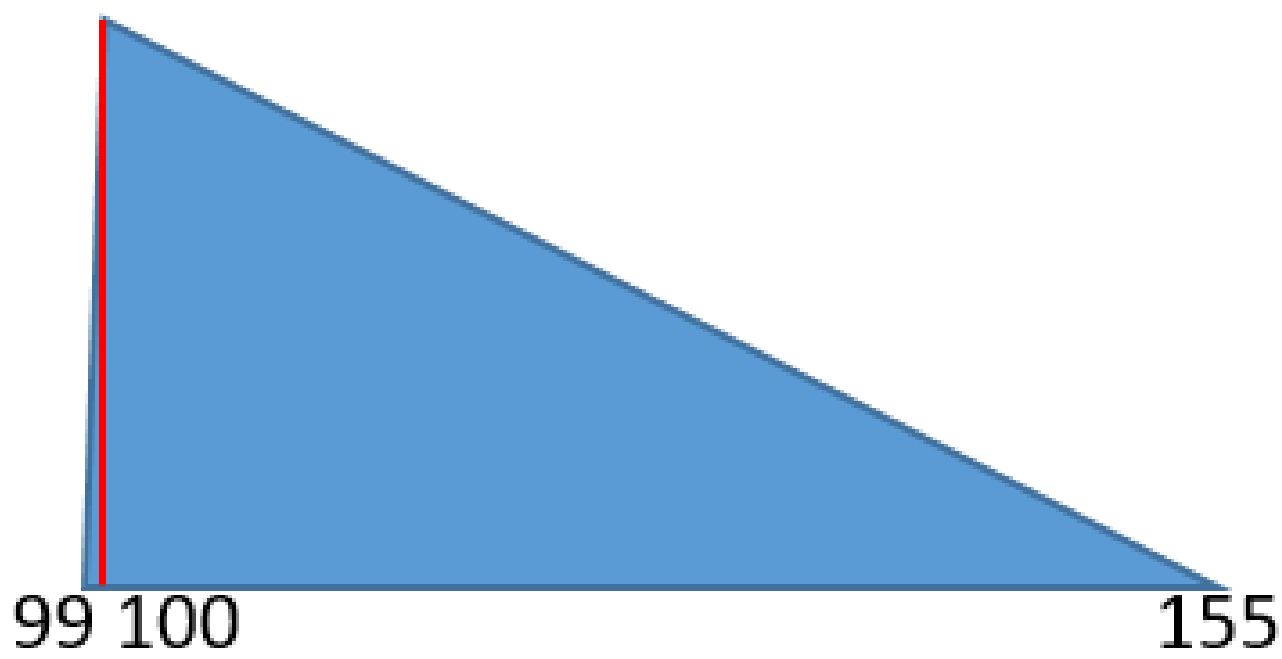

However, the project realized that this high uncertainty distribution allows for elements to have a greater probability of having lower values (70\%) than that of the low uncertainty set $(90 \%)$, as can be seen in Figure 8 .

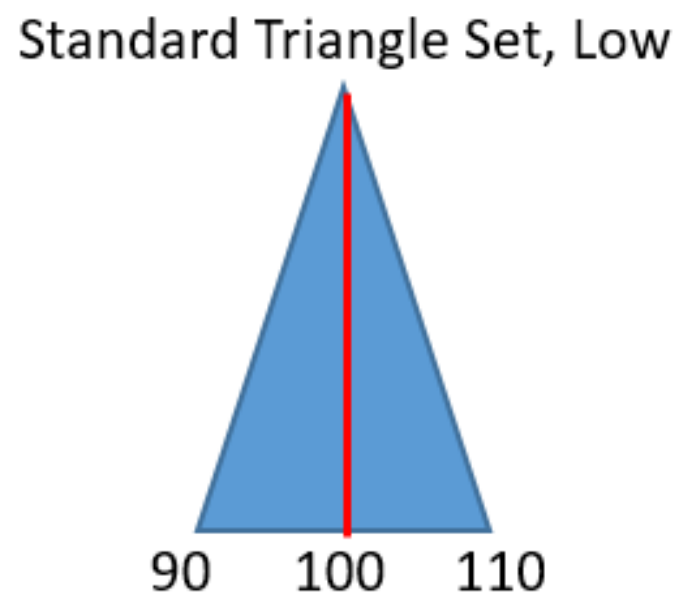

\section{Figure 8. Standard Triangular Distribution for Low Uncertainty}

The low end of the high uncertainty is $70 \%$ versus the $90 \%$ on the low end of the low uncertainty.

With that in mind, a conservative approach was taken where the project implemented new conservative distributions. Here, in Figure 9, is an example of the new high uncertainty triangular distribution (for both duration and TI cost $\mathrm{U}$ ).

\section{Figure 9. Conservative Triangular Distribution for High Uncertainty}

Moreover, the previous high uncertainty with values of 70 , 100,130 is now $99,100,155$. Now, high risk items will now only have a $1 \%$ probability of falling below the point estimate, while having a $55 \%$ probability of going beyond the point estimate. 


\section{MPDR JCL MODEL RESULTS}

\section{1 mPDR Summary of Results}

Now that the Analysis Schedule was complete with duration uncertainties, cost uncertainties, risks, and costs to date, the project was ready to get the JCL results. Performing the mPDR JCL involved running 3500 Monte Carlo simulations, or probability simulations, to produce the scatterplot seen in Figure 10.

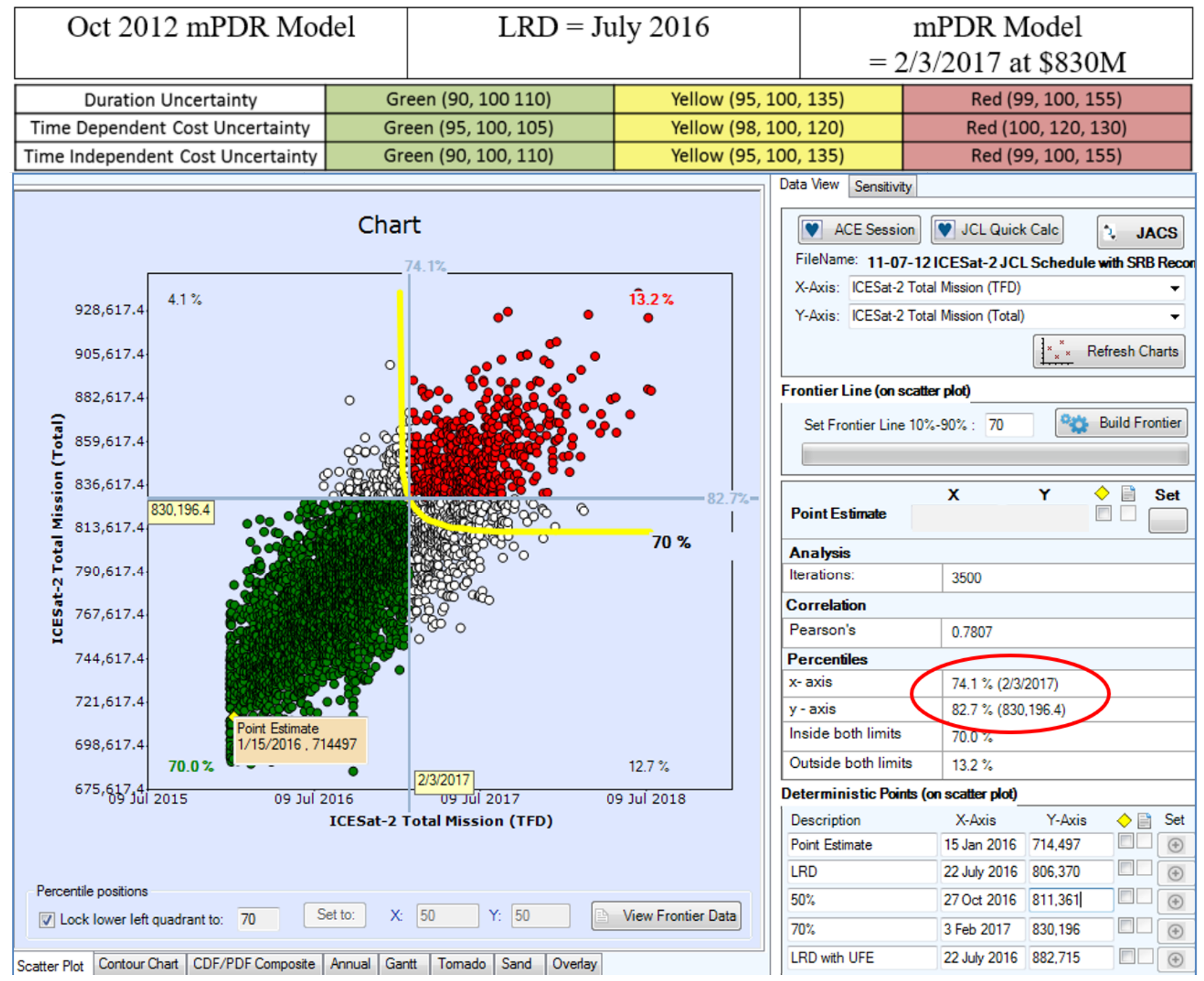

Figure 10. mPDR JCL Results

Each scatterplot dot represents a specific result from the simulation calculation. Therefore, within this shotgun blast there are 3500 iterations of cost and schedule risk analysis. The $\mathrm{x}$-axis represents the final completion date and the $\mathrm{y}$-axis represents the final cost.

The yellow frontier curve specifies all the cost/schedule combinations that will meet the $70 \%$ joint confidence of cost and schedule. The project selected this point on the knee of the curve, as it's recommended to be as close as possible to the center of the cluster.

Recapping the results, with $70 \%$ confidence, the LRD would be February 2017 at $\$ 830 \mathrm{M}$. This was about 12 months beyond our point estimate, which was launch date minus the 6 months of margin we were holding at the time.

The mPDR JCL results were consistent with previous parametric cost and schedule estimates.

These results came from many adjustments of the model, to yield results that the project management team agreed with. During the testing period, we had learned that uncertainty distributions drive the schedule durations much more than discrete risks or cost uncertainty. Moreover, duration uncertainty impacts the results much more than discrete risks, as duration uncertainty impacts the entire model, if applying an overall uncertainty to all work elements, while the discrete 
risks will impact only specific elements and their subsequent downstream elements.
The mPDR results were able to produce a list of schedule uncertainty drivers, which indicate the elements that influenced the model, as seen in Figure 11.

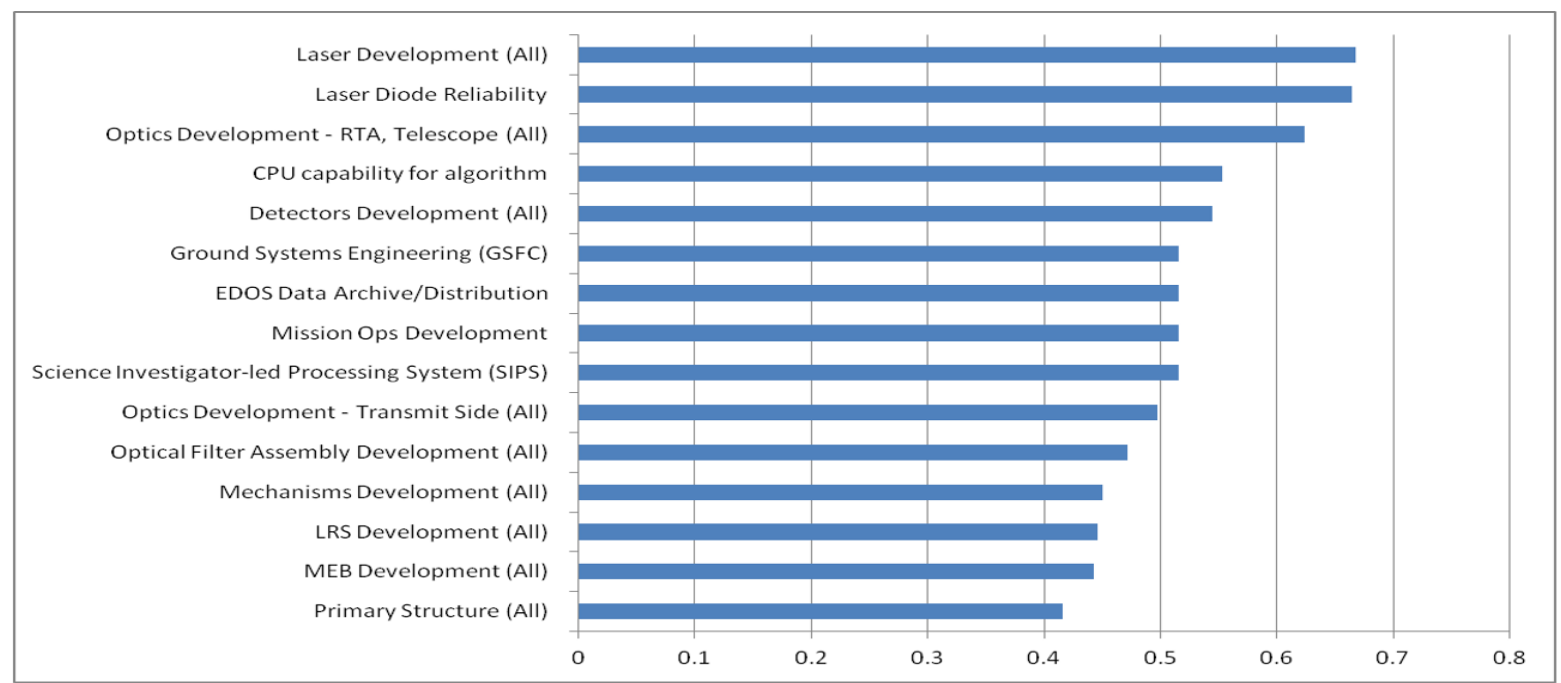

Figure 11. Schedule Uncertainty Drivers

Similarly, the model was able to provide a list of the discrete risks that would appear on the critical path of the model, indicating that these items were the top drivers of the analysis schedule. This complete list can be seen in Figure 12.

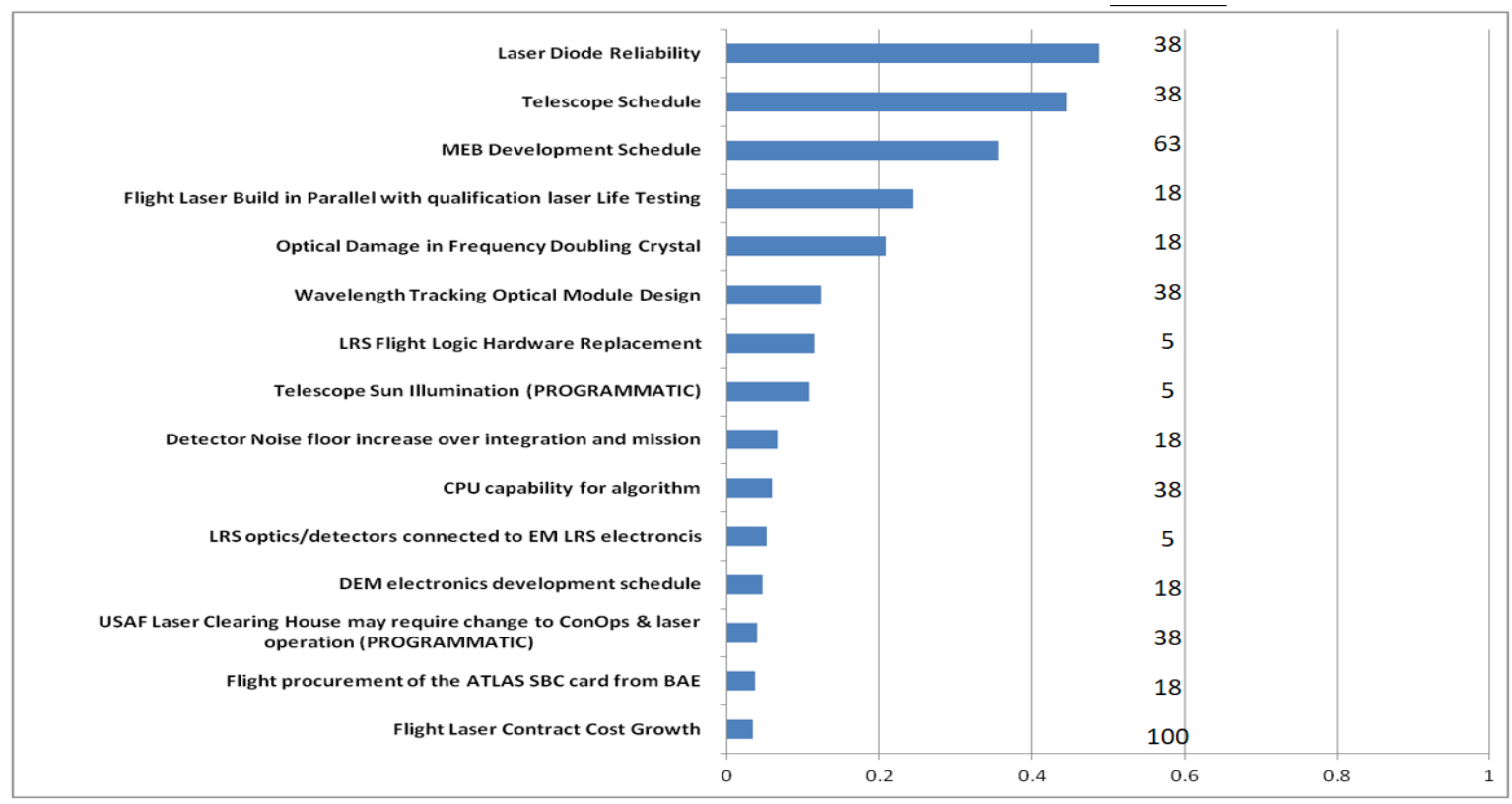

Figure 12. Schedule Risk Drivers

\section{SCHEdule Slip ANd Model AdJUSTMENTS}

The year following the mPDR, the project had sustained a one-year slip in the LRD due to problematic systems engineering requirement issues which impacted all project subsystems. In short, the project team was not making progress towards their critical design.
This slip moved the project planned LRD from July 2016 to July 2017, which was an additional 5 months beyond the 2012 model's 70\% JCL result for the LRD of February 2017.

As the project was quickly approaching $\mathrm{mCDR}$, the need for reliable JCL results increased significantly.

The project held discussions on the JCL modeling process and focused on the input uncertainty distributions. 
Specifically, to identify the uncertainty distributions that the 2012 mPDR model would have needed to produce a $70 \%$ LRD result of July 2017. The project wanted to use past performance and historical data from the schedule slip to improve our parametric model.
This led the project to develop uncertainty distributions that incorporated the project's past performance and historical data to improve our parametric model.

Figure 13 shows how the slip looked when plotted on a timeline.

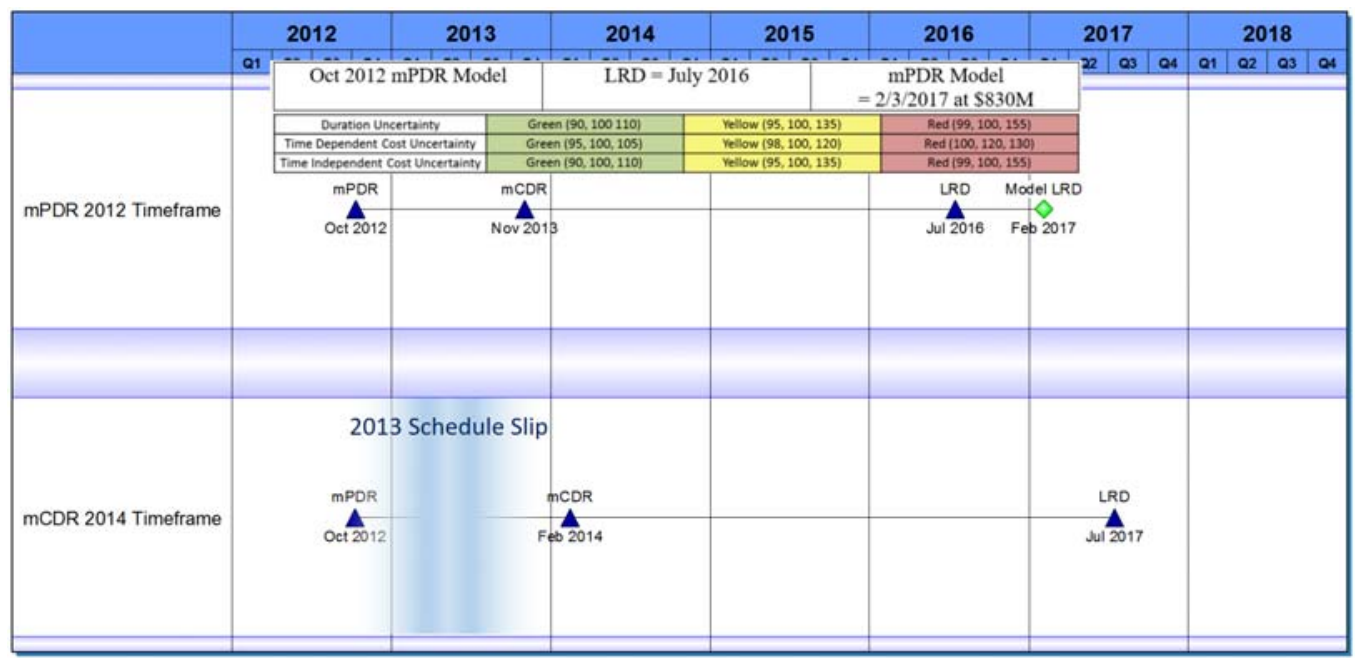

Figure 13. 2013 Schedule Slip

The top row is the schedule as it was during the mPDR timeframe and the second row is the MCDR timeframe. The blue milestones were the actual milestone dates from the schedules. The green diamond is the model projection.

Looking at the mCDR timeframe, the blue area, 2013 schedule slip, indicates the timeframe of the slip. Also, one can see how the one-year slip, from July 2016 to July 2017 , jumped right over our model LRD.

Obviously the model needed to be updated and improved with better data, derived from this post-slip knowledge.
Moreover, the project needed to identify the uncertainties that should have been included in the mPDR model, to yield a $70 \%$ confident LRD of July 2017.

Therefore, after many adjustments within the model, the project increased the uncertainties for the top schedule drivers in the model to reach the LRD of July 2017 at $\$ 946 \mathrm{M}$.

A new color coding system was developed to easily see the changes in uncertainties between models, as can be seen in Figure 14. Green changed to yellow. Yellow changed to red. Red changed to black.

Oct 2012 mPDR JCL Uncertainties:

\begin{tabular}{|c|c|c|c|}
\hline \multicolumn{2}{|c|}{ Oct 2012 mPDR Model } & LRD = July 2016 & \multicolumn{2}{c|}{$\begin{array}{c}\text { mPDR Model } \\
=2 / 3 / 2017 \text { at } \$ 830 \mathrm{M}\end{array}$} \\
\hline \hline Duration Uncertainty & Green $(90,100110)$ & Yellow $(95,100,135)$ & $\operatorname{Red}(99,100,155)$ \\
\hline Time Dependent Cost Uncertainty & Green $(95,100,105)$ & Yellow $(98,100,120)$ & $\operatorname{Red}(100,120,130)$ \\
\hline Time Independent Cost Uncertainty & Green $(90,100,110)$ & Yellow $(95,100,135)$ & $\operatorname{Red}(99,100,155)$ \\
\hline
\end{tabular}

The Oct 2012 mPDR Model, needed the following uncertainties in order to yield a 70\% confident LRD of July 2017 at \$946M:

\begin{tabular}{|c|c|c|c|}
\hline $\begin{array}{c}\text { Oct } 2012 \text { mPDR Modified } \\
\begin{array}{c}\text { Model } \\
\text { w/ Post Knowledge } \\
\text { Uncertainties }\end{array}\end{array}$ & LRD = July 2016 & $\begin{array}{c}\text { Oct } 2012 \text { mPDR Model w/ } \\
\text { Post Knowledge Uncertainties } \\
=7 / 21 / 2017 \text { at \$946M }\end{array}$ \\
\hline \hline Duration Uncertainty & Green $(90,100110)$ & Yellow $(95,100,135)$ & Red $(99,100,155)$ \\
\hline Time Dependent Cost Uncertainty & Yellow $(98,100,120)$ & Red $(100,120,130)$ & Black $(180,210,240)$ \\
\hline Time Independent Cost Uncertainty & Green $(90,100,110)$ & Yellow $(95,100,135)$ & Red $(99,100,155)$ \\
\hline
\end{tabular}

Tri $(200,220,240,10,90)$ replaced the schedule duration uncertainty for the following discrete risks:

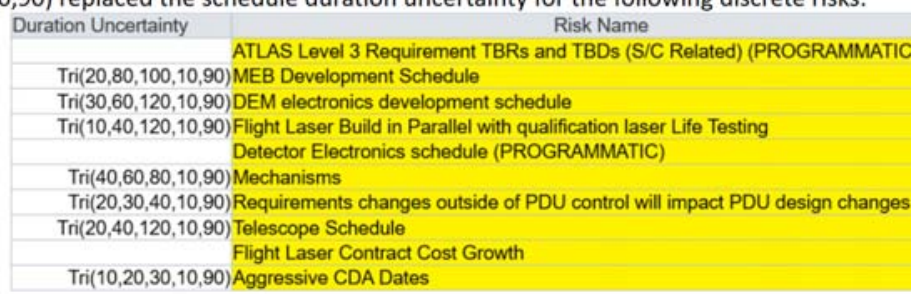

Tri $(10,20,30,10,90)$ Aggressive CDA Dates

Figure 14. 2013 Schedule Slip Model Adjustments 
The October 2012 mPDR model, modified with this postknowledge information, needed these new October 2012 mPDR modified model with post-knowledge uncertainties, to yield a 70\% confident LRD of July 2017.

Black was applied to the laser subsystem development, ATLAS integration and test (I\&T), and observatory I\&T. We wanted to increase the uncertainties so that the model date and cost would be equal to our current estimate. The rationale for this decision was based on the laser subsystem, which was the new technology and historical schedule driver.
Then, the project aggressively increased the schedule duration uncertainty for the discrete risks that were related to developmental issues. This approach is known as a datadriven method or a performance method to updating a model.

Figure 15 shows that PDR uncertainties in the mPDR timeframe needed the updated uncertainties in the mCDR timeframe to move the model LRD from February 2017 to July 2017, shown at the orange diamond.

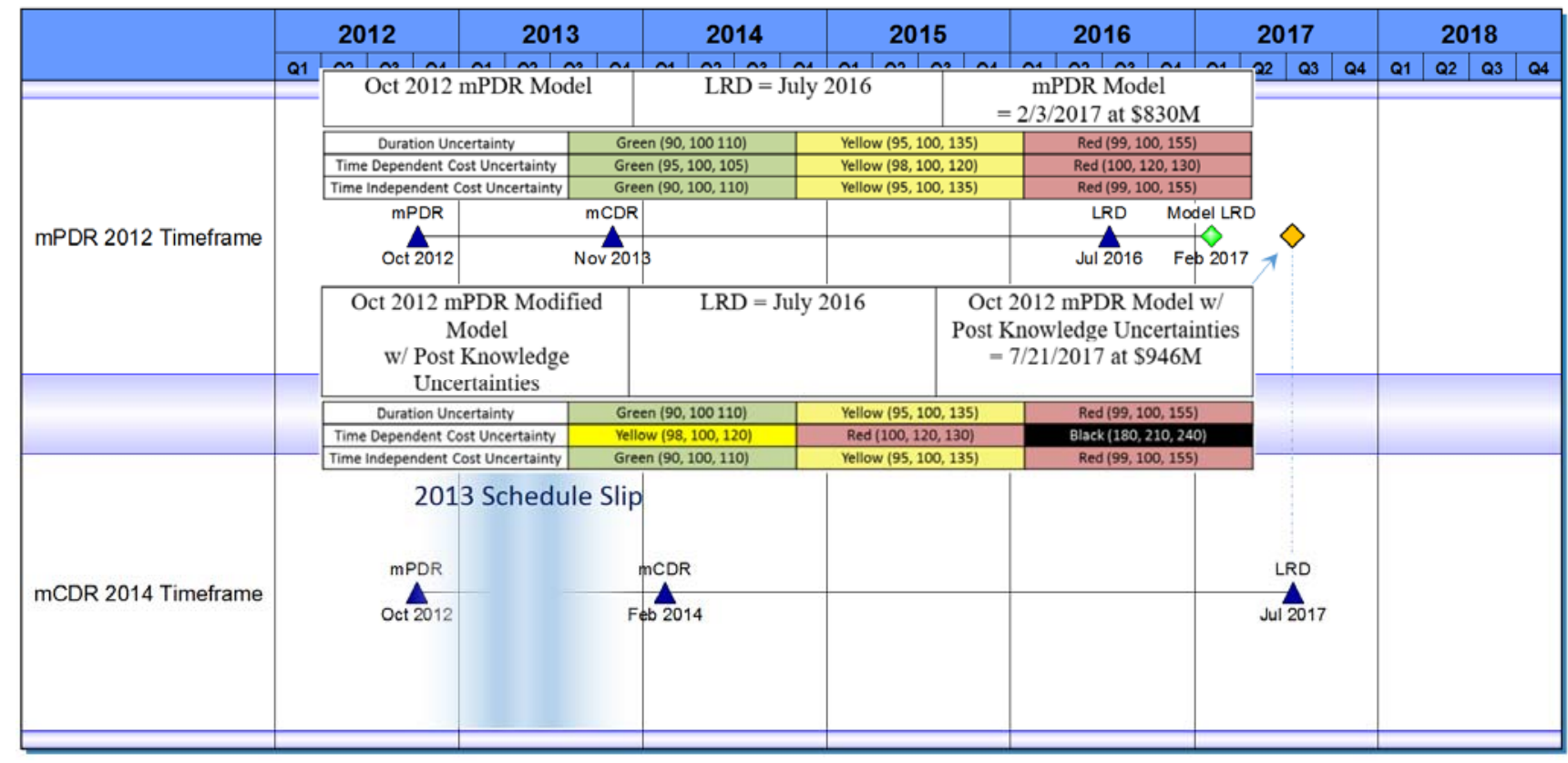

Figure 15. Uncertainty Adjustments

The project increased the time-dependent cost uncertainty to align with the new costs. However, the project did not modify the duration uncertainty nor the time-independent cost uncertainty as we wanted the discrete risks associated with the cause of the slip (i.e., having developmental schedule issues), to push out the model date.

\section{MCDR JCL MODEL RESULTS}

Now that our uncertainties were updated to reflect the project's past performance, the final step involved creating a new February 2014 mCDR Analysis Schedule to reflect the new one-year slipped IMS and new LRD of July 2017. These new post-knowledge uncertainties were then applied to this new February 2014 mCDR model with the new costs and actuals (for February 2014) and the new LRD of July 2017.

Again running 3500 simulation iterations, the February 2014 mCDR model results informed that with $70 \%$ confidence, the ICESat-2 mission would launch in August 2018 with a cost of $\$ 1,044$, as seen in Figure 16. 


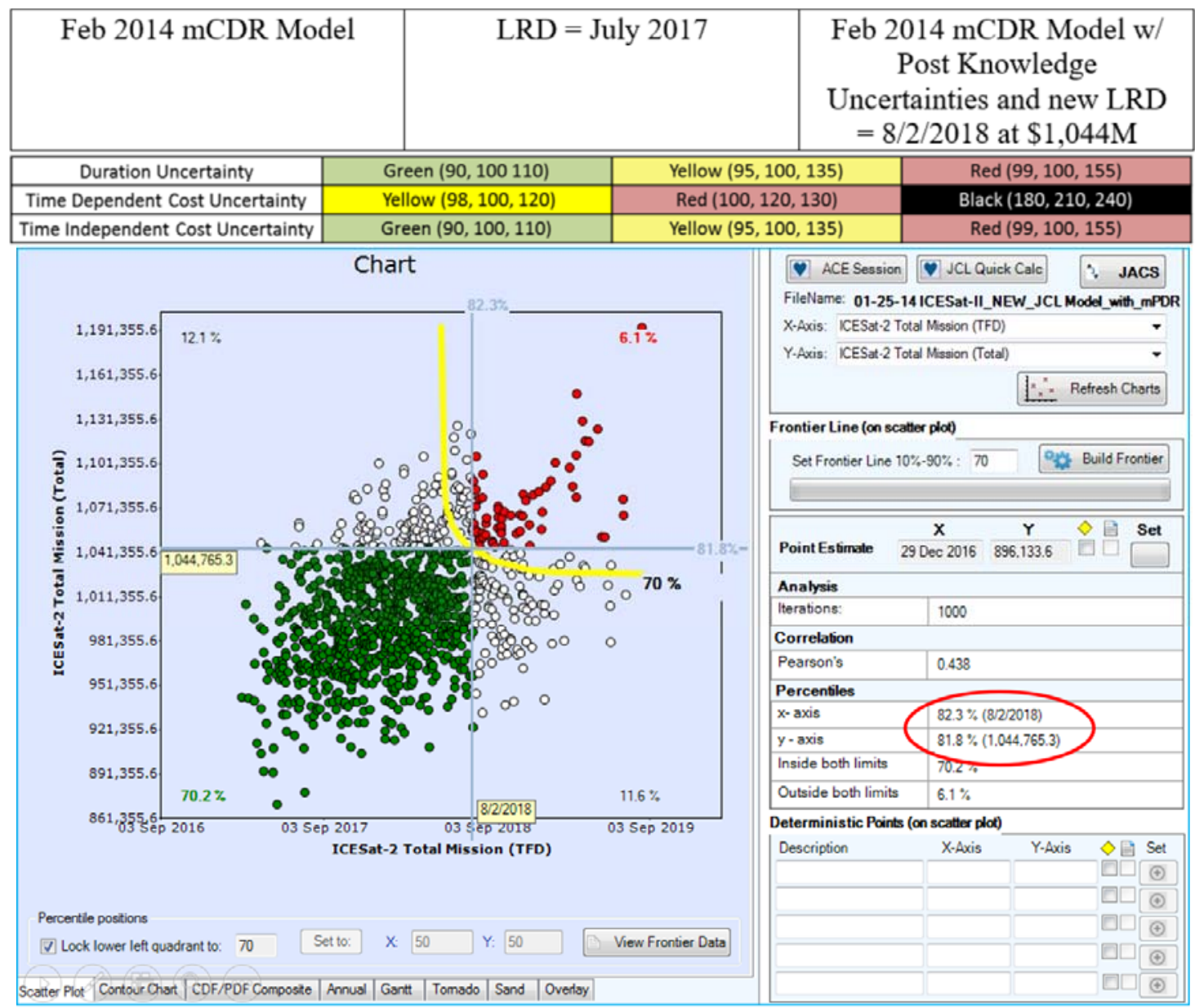

Figure 16. mCDR JCL Results

This February 2014 mCDR model informed that the slip would be due to a laser failure, the top schedule driver within the model.

The mCDR model shifted the LRD from July 2017 to August 2018 for a total of 13 months.
Plotting this mCDR result of August 2018 on the mCDR timeline allows for easy comparison to the mPDR results, as seen in Figure 16. With these new post-knowledge uncertainties applied to our new model, we see the result on the mCDR timeline, in the green diamond.

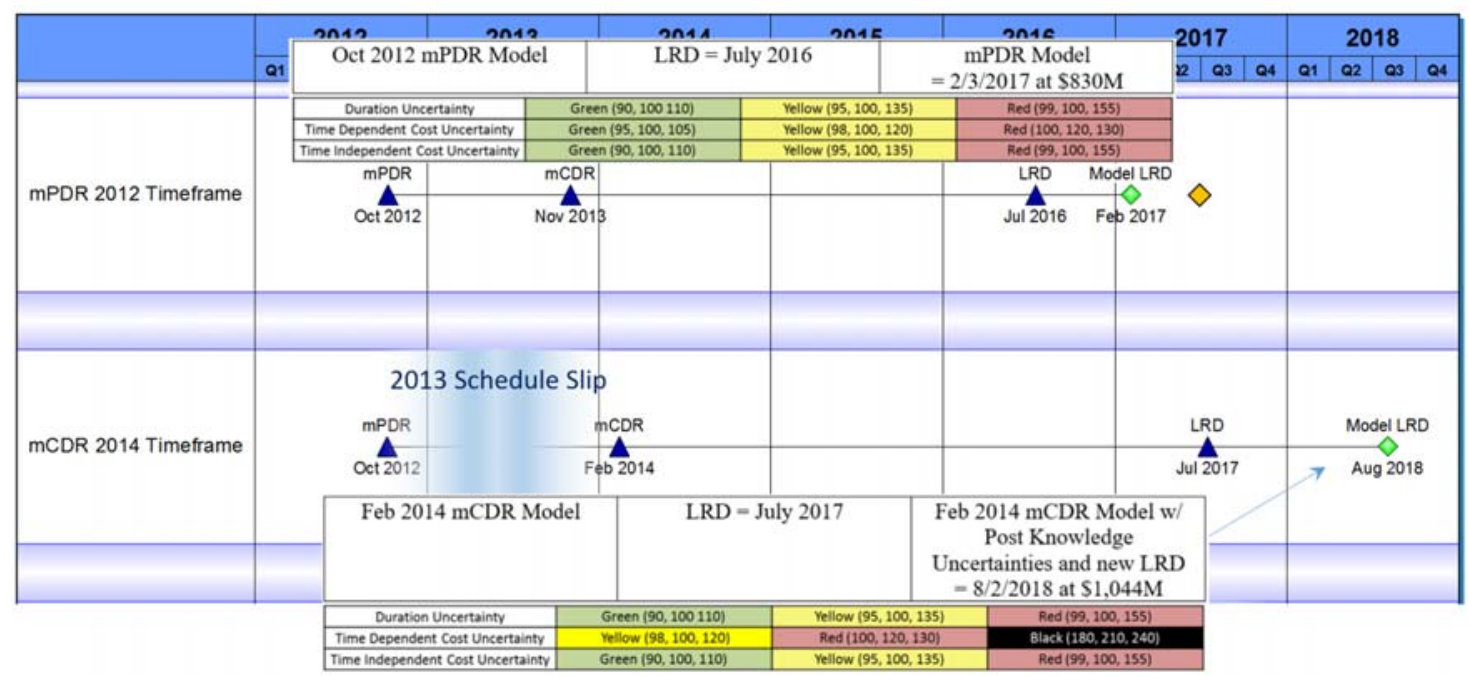

Figure 17. Results of $m P D R$ compared to the $m C D R$ 


\section{MCDR RESULTS \& THE ICESAT-2 LAUNCH}

ICESat-2 successfully launched on September 15, 2018 with a PM agreement of \$1B and \$56M.

Recapping the model's results, the project's mCDR model from $4-1 / 2$ years ago, suggested that with $70 \%$ confidence, the ICESat-2 LRD would move from July 2017 to August 2018 and would be due to the laser subsystem.

Adding an additional row to the comparison chart and looking at the ICESat-2 final schedule row in Figure 17, we can see how the model nearly anticipated the 2017 schedule slip.

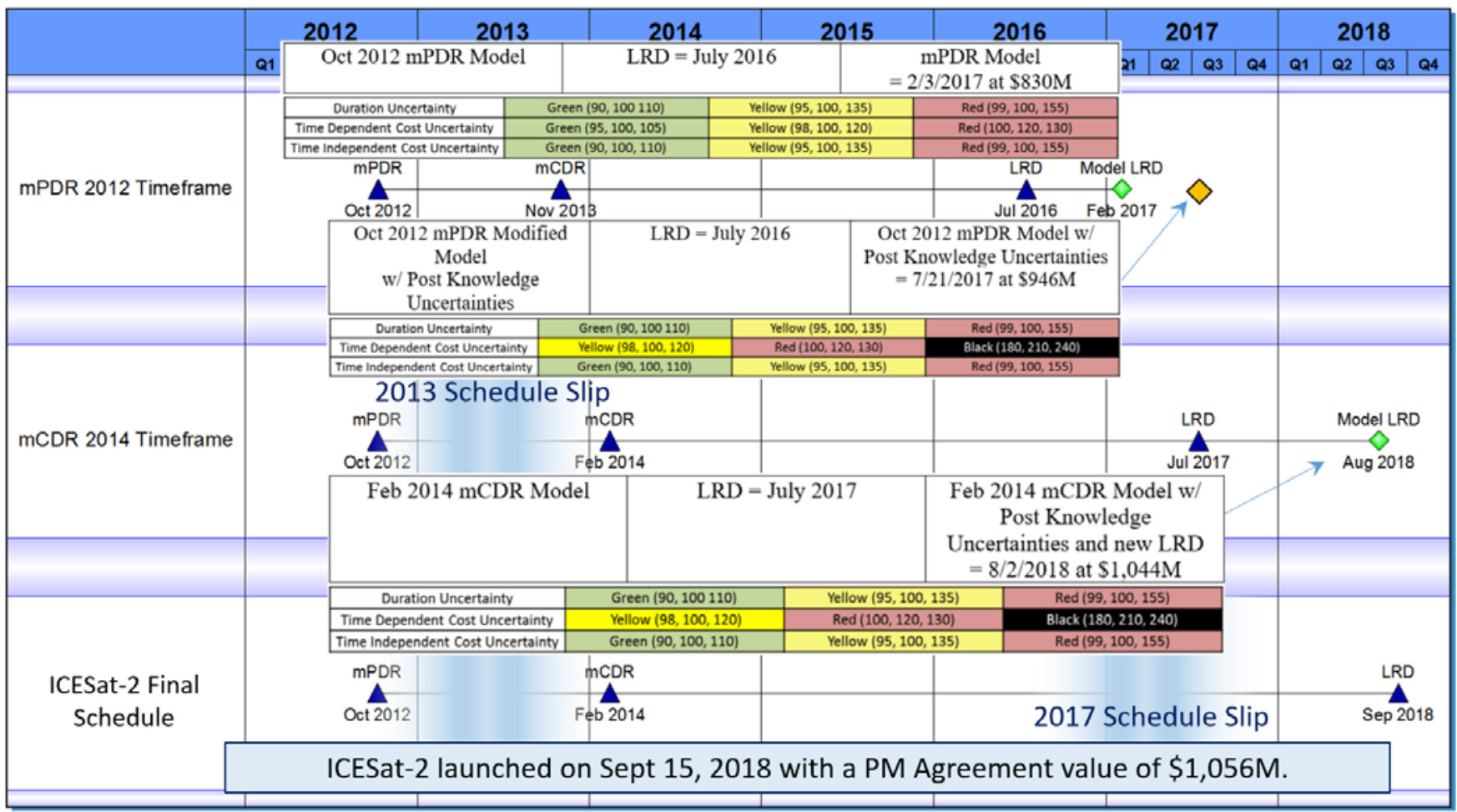

Figure 18. mCDR Results with the ICESat-2 LRD

Addressing this 2017 schedule slip, the project had a laser failure, which was the highest discrete risk and uncertainty driver within the model.

The laser failure was due to an anomaly that hadn't been seen before. Intermetallic growth between the Gold and Indium in the housing caused the laser slab crystal to fracture. This fracture was attributed to new laser technology, involving the variable energy laser. Lowering the laser energy actually causes more heat and a faster chemical reaction between the Gold and Indium. To be clear, Gold and Indium have been used on many laser missions to date without an issue. This was a true anomaly that had previously never occurred on a laser.

The laser fell to the dreaded "unknown unknown." The team had to fabricate new lasers using different materials. This fix pushed out the project an entire year.

\section{SUMMARY AND LESSONS LEARNED}

The goal of this paper is to provide insight that an advocate JCL can predict and quantify issues on new technology missions.
The information provided supports the notion that projects should revisit their parametric models on a recurring basis so the project, and all stakeholders, have a better understanding of the probability of completion.

In the instance of ICESat-2, the 2013 schedule slip was the impetus to revisit and update the model. However, a project does not need a slip to occur to update their model. A project could continuously improve their parametric models using various scheduling performance metrics, or integrate the project's earned value management performance data into the model.

The majority of work and effort has already been accomplished in the setup of the original model. The analyst would need to update the analysis schedule to align with the current IMS, update the performance data for the uncertainties, as well as the cost actuals and risk register.

In conclusion, this illustrates how a parametric model can be continuously improved to produce valuable results for a project, even in cases of LRD delays. 
APPENDICES

\section{A. MPDR RESUlts: Risk AdJUSTED ANNUAL COST}

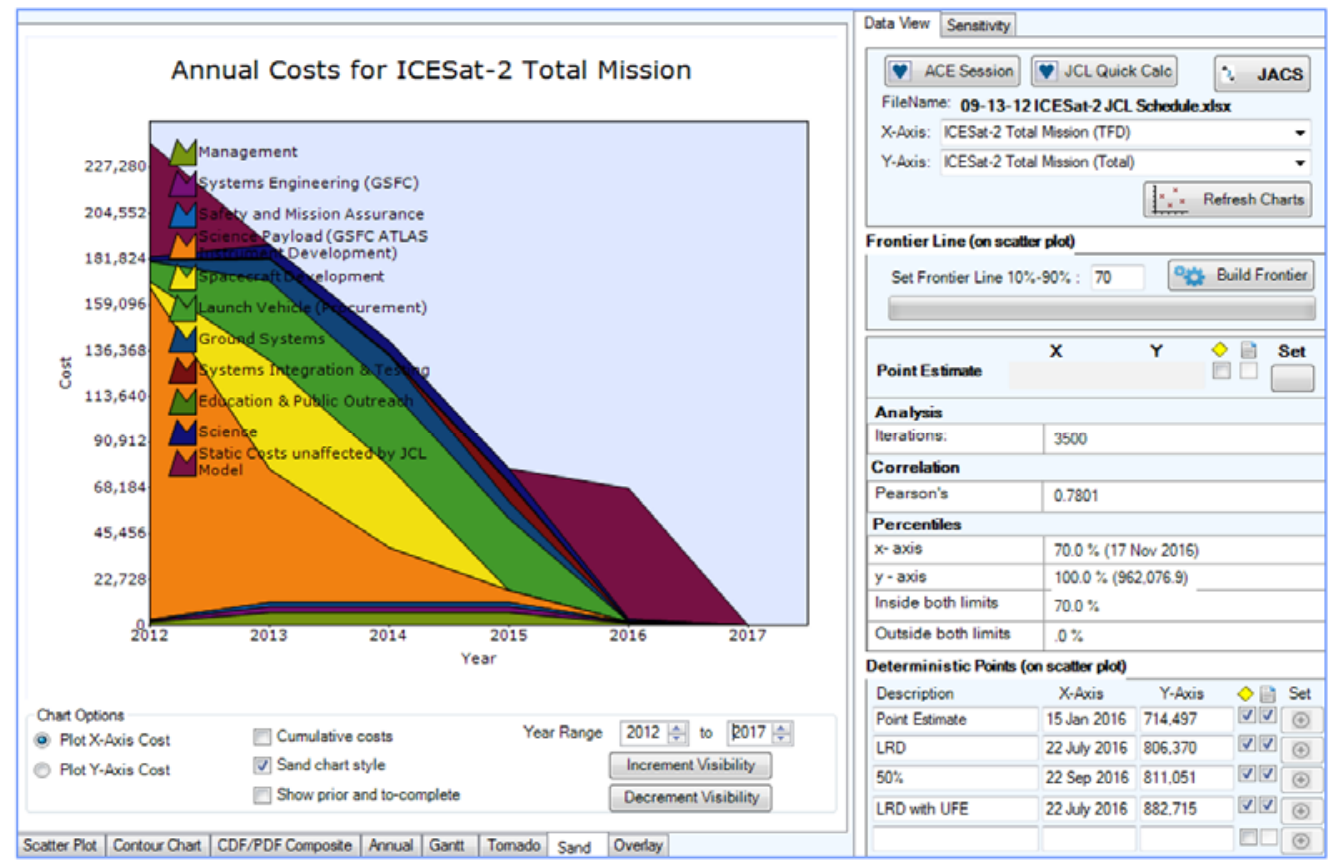

\section{B. MPDR RESUlTs: COST S-CuRVE}

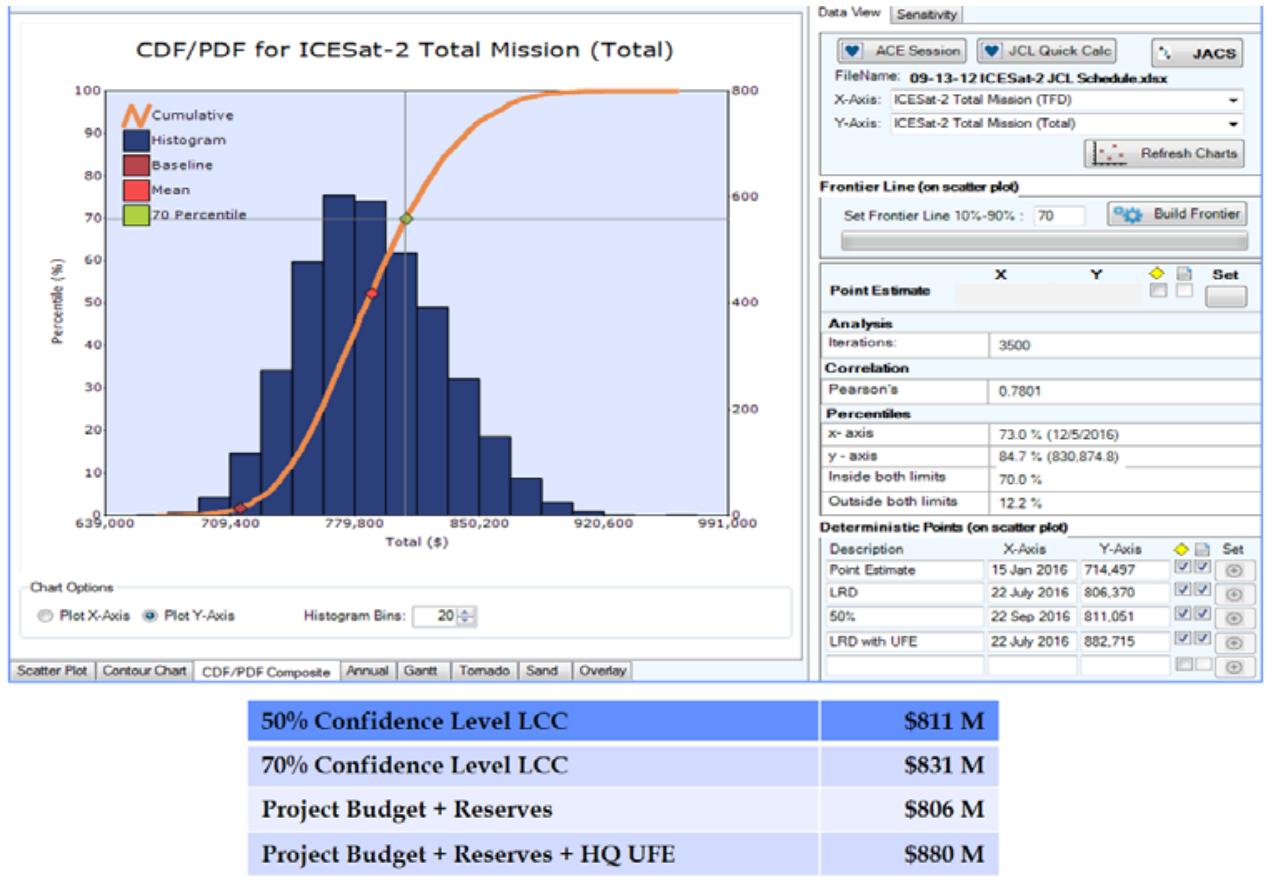




\section{MPDR RESULTS: FINISH DATE S-CURVE}

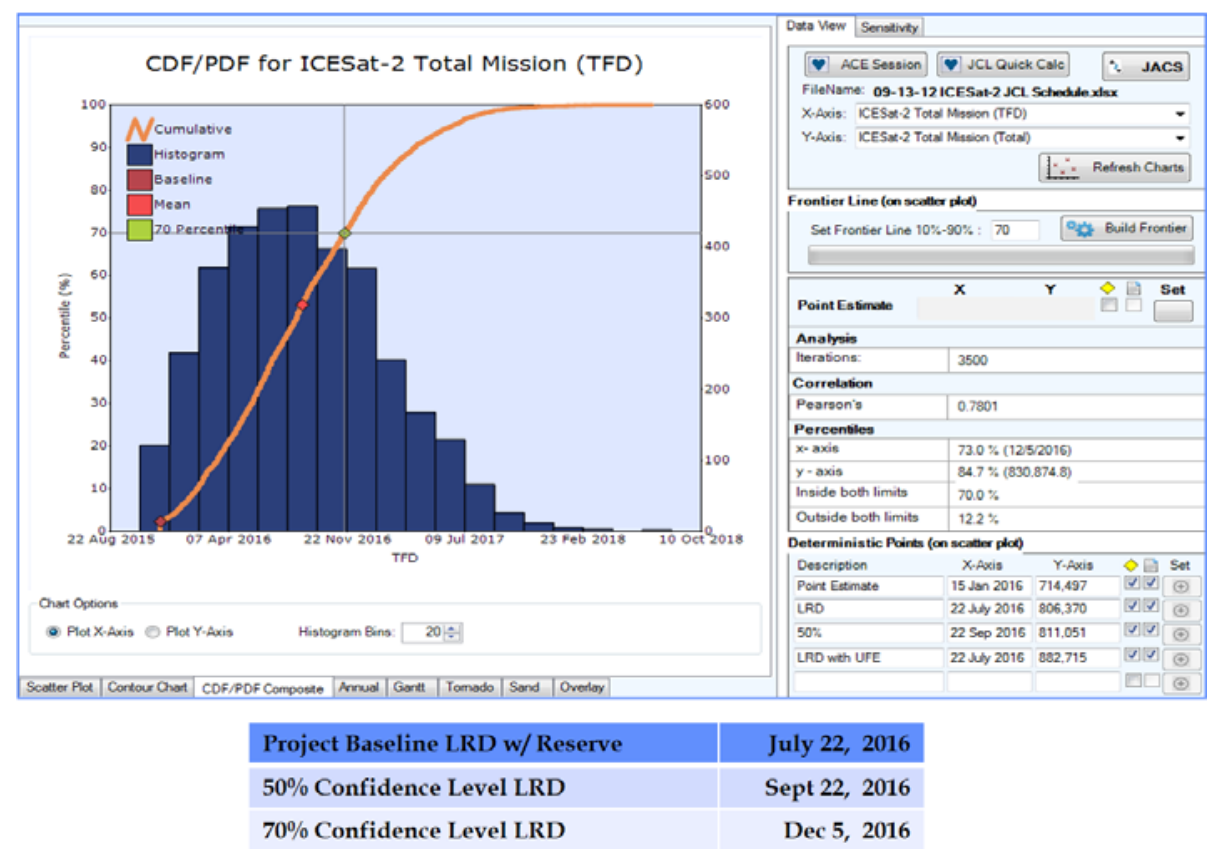

\section{ACKNOWLEDGEMENTS}

I'd like to thank all of my NASA Customers who provided me the opportunity to head up the JCL initiative on ICESat2. Special thanks to:

James Johnson, NASA HQ Office of the Chief Financial Officer

Mark Seidleck, NASA Goddard Space Flight Center, ICESat-2 Deputy Project Manager, Resources

Param Nair, NASA Goddard Space Flight Center, Resource Analysis Office

\section{REFERENCES}

[1] John Neatrour, MCR: SCEA Paper "Methods for Parametric Joint Cost \& Schedule Risk Analysis"

[2] Stephen A. Book, MCR: "Quantifying the Relationship Between Schedule and Cost" Winter 2007-2008 http://www.scribd.com/doc/7106935/Quantifiying-therelationship-between-schedule-and-cost

[3] Elliott/Rippe/Hogan: SCEA Conference 2011, Best Paper Research Track "Joint Cost Schedule Model (JCSM) Recent AFCAA Efforts to Assess Integrated Cost \& Sched Analysis" http://www.sceaonline.org/awards/papers/2011_research.pdf

\section{BIOGRAPHY}

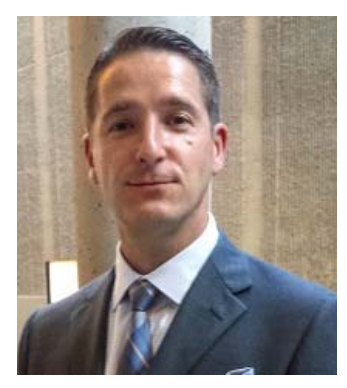

Joseph Krygiel After receiving his Bachelor of Science in Industrial Engineering from Penn State University in 1999, he has supported various customers and clients at the NASA Goddard Space Flight Center, holding an array of positions.

His career began as a Mechanical Systems Integration Engineer, where he managed spaceflight hardware for the Hubble Space Telescope. Over the years, he progressed to Project Engineer, to now providing Analytical Project Management Consultation. He served as the Planning and Schedule Manager on ICESat-2 for the entire life cycle of the mission, culminating to more than 10 years of service, on one project alone.

He currently supports NASA Headquarters as the Agency Scheduling Community of Practice ( $\mathrm{SCoPe}$ ) representative for NASA GSFC, the GSFC Earth Science Projects Division as the Program Planner, as well as an Integrated Program Team Leader supporting the Program Analysis and Control contract. His current positions allows him to manage both at the project and program levels through the use of process planning and controls management, while at the same time, allowing him to develop new methods of facilitating NASA Agency communication. 